\title{
Transoral Robotic Thyroidectomy: The Overview and Suggestions for Future Research in New Minimally Invasive Thyroid Surgery
}

\author{
Jeong Min Choo, M.D., Ji Young You, M.D., Ph.D., Hoon Yub Kim, M.D., Ph.D., FACS \\ Department of Surgery, KUMC Thyroid Center, Korea University Hospital, Korea University College of Medicine, Seoul, Korea
}

Purpose of review: This journal introduces a overview in depth about the evolution of transoral robotic thyroidectomy, which has been encountering major confrontations in expanding its indications.

Recent findings: Transoral robotic thyroidectomy (TORT) is one of the newest approaches and draws attention because of its cosmetic excellence. The major advantage of TORT is comparatively smaller flap dissection area than other remote-access methods. The other advantage of TORT is that the wounds of lips fades out over time, and leaves concealed scar near axilla.

Summary: TORT can be done safely to the appropriately selected patients by surgeon expertise in robotic thyroidectomy. It might be a potential alternative surgical approach for thyroidectomy to surgeons who are experienced in remote-access robotic surgery.

Keywords: Robotic surgical procedure, Oral cavity, Thyroidectomy, Thyroid nodule, Thyroid neoplasm

This is an Open Access article distributed under the terms of the Creative Commons Attribution Non-Commercial License (http:I/ creativecommons.org/licenses/by-nc/4.0/) which permits unrestricted non-commercial use, distribution, and reproduction in any medium, provided the original work is properly cited.
Received February 19, 2019

Revised March 8, 2019

Accepted March 12, 2019

Corresponding author

Hoon Yub Kim

Department of Surgery, Korea

University College of Medicine, 73

Inchon-ro, Seongbuk-gu, Seoul

06966, Korea

Tel: +82-2-920-6849

Fax: +82-2-928-1631

E-mail: hoonyubkim@korea.ac.kr ORCID:

http://orcid.org/0000-0002-6731-3912

Jeong Min Choo and Ji Young You are co-first authors who have contributed equally to this article.

Copyright @ 2019 The Journal of Minimally Invasive Surgery. All rights reserved.

\section{INTRODUCTION}

Thyroidectomy is the standard of the treatment for patients with organ-confined thyroid cancer and with benign thyroid disease. Thyroidectomy has dramatically changed since the introduction and development of minimally invasive techniques during last 20 years. $^{1-3}$ Advantages and disadvantages of endoscopic thyroidectomy are still under the debate. Some argue that it is a distrustful approach, considering that there have been only small number of cases, limited indications, more surgical trauma, more extensive flap dissection, longer operation time and technical difficulties. However, others claim that despite these drawbacks, some advantages over- come those difficulties. ${ }^{4,5}$

In fact, techniques in minimally invasive surgery have constantly advanced over time. ${ }^{6}$ In order to improve outcomes of endoscopic thyroidectomy, novel approaches, new instruments, robotics and retraction methods have devised, applied, and upgraded. The aim of such evolution is to improve the cosmetic result, and also to reduce the peri-operative complications. ${ }^{7.8}$ Therefore, as a part of such effort, surgeons vigorously tried a new endoscopic method, such as transoral vestibular approach, which hardly leaves noticeable scars. ${ }^{9}$

Robotic thyroidectomy itself has been a challenge for thyroid surgeons. The challenges start from different ways of approach: directly-on-neck, transaxillary and transareolar. ${ }^{10}$ With the ad- 
vent of transoral vestibular approach, robotic surgery has come up with surprising remarkable results. ${ }^{11-15}$ Among those various approaches, transoral robotic technique has opened a novel generation of minimally invasive thyroid surgery and concomitant expansion of new instrumentation, and technology. ${ }^{16}$

The development includes the effort to overcome the proximity of the endoscopic instruments, elimination of triangulation, tremor, internal and external crashes among instruments, restriction of movement and access to the structures. ${ }^{17,18}$

Here, we discuss the development of robotic surgery and also compare the transoral robotic thyroidectomy (TORT) with conventional and endoscopic thyroidectomy techniques.

\section{INDICATIONS AND CONTRAINDICATIONS}

A strict and careful selection of patients is the most important factor to ensure a good outcome in TORT. Recently, due to its full accessibility to the contralateral lobe and central compartment, robotic surgery has been extended to total thyroidectomy with central lymph node clearance for patients with thyroid cancer. ${ }^{19}$ One of the greatest limitations is the size of the nodule. Despite the limitations, as the experience accumulates, criteria of inclusion and exclusion have been expanded, advanced disease could be candidate to this approach.

Indications for TORT can be benign thyroid nodule, such as a large thyroid cyst, single-nodular goiter, or multinodular goiter (single/largest nodule $<6 \mathrm{~cm}$ in size on preoperative ultrasound); and also papillary carcinoma $(<4 \mathrm{~cm}$ in size and without evidence of extensive lymph node metastasis on preoperative ultrasound) and follicular neoplasm (Table 1).

Absolute contraindications for TORT are the patients who are unfit for surgery, and who cannot tolerate general anesthesia. Poorly differentiated thyroid cancer, undifferentiated thyroid cancer; locally advanced thyroid cancer with tracheal/ esophageal invasion; and thyroid cancer with N1b lymph node involvement; or with posterior extrathyroidal extension should also be excluded. And the patients who have previous history of neck surgery, radiation, and of oral abscess should be excluded (Table 1).

\section{PREOPERATIVE REQUIREMENTS}

As in all endoscopic procedures, TORT also requires good preoperative planning. Preoperative factors include a thorough preoperative work-up, dental care and chlorohexidine mouth wash to maximize oral hygiene, laryngoscopic assessment of vocal cord, administration of prophylactic antibiotics (amoxicillin / clavulanate) and inspection of instrumentation.

Thorough preoperative work-up includes a thyroid ultrasonography and/or CT scan of neck. All patients should receive an ultrasound guided fine needle aspiration biopsy before surgery to plan the appropriate procedure. It is important to obtain informed consent from all patients, including a novelty of this procedure and associated risks. In addition, the informed consent must include the fact that the da Vinci robot is not approved by the Food and Drug Administration (FDA) for this approach, yet.

Since the transoral surgery is clean-contaminated surgery, the approach increases the risk of infection of the anterior neck region. The three vestibular incisions create a new communication between the oral cavity and neck. Hence, during neck exploration, oral microflora can spread, originally an aseptic operation can become a infectious surgical intervention. ${ }^{20}$ It is also important to prepare every single necessary instrumentation in the preoperative setting.

\section{PROCEDURES}

\section{Working space formation}

Under general anesthesia, the patient lies down in supine position. After intubation with nerve monitoring endotracheal electromyogram tube, the patient's neck is slightly extended. Three incisions are made in the gingival-buccal sulcus; one

Table 1. Indications and contraindications for transoral robotic thyroidectomy

\begin{tabular}{ll}
\multicolumn{1}{c}{ Indications } & \multicolumn{1}{c}{ Contraindications } \\
\hline - Benign thyroid nodule, such as a large thyroid cyst, single-nodular & - Patients unfit for surgery and who cannot tolerate general anesthesia \\
goiter, or multinodular goiter (single/largest nodule $<6 \mathrm{~cm}$ in size & - Poorly differentiated thyroid cancer \\
on preoperative ultrasound) & - Undifferentiated thyroid cancer \\
- Papillary carcinoma $(<4 \mathrm{~cm}$ in size and without evidence of & - Locally advanced thyroid cancer with tracheal/esophageal invasion \\
extensive lymph node metastasis on preoperative ultrasound) & - N1b lymph node involvement \\
- Follicular neoplasm & - Posterior extrathyroidal extension \\
& - Previous history of neck surgery \\
& - Previous history of neck radiation \\
& - Presence of oral abscess
\end{tabular}


in the midline, a $1.5 \mathrm{~cm}$-length inverted $\mathrm{U}$ shape incision approximately $2 \mathrm{~cm}$ above the frenulum labii inferioris, and two $0.5 \mathrm{~cm}$-length incisions laterally near the angle of mouth (Fig. 1A). The central incision is made first. Once the periosteum is identified through the midline incision, a submental subplatysmal pocket is formed by blunt dissection with mosquito and Kelly forceps to create a tunnel from the edge of the mandible to lower neck by injecting 1:200,000 epinephrine saline mixture for hydro-dissection of the subplatysmal plane. Then, blunt dissection with $8 \mathrm{~mm}$-tipped vascular surgical tunneler is gently performed to elevate the platysma off the strap muscles all the way down towards the suprasternal notch (Fig. $1 \mathrm{~B})^{20}$

After the creation of adequate flap, the endoscope cannula is inserted. $\mathrm{CO}_{2}$ insufflation $(5 \sim 7 \mathrm{mmHg})$ is introduced and maintained via the central port. A similar blunt dissection is also performed through the two lateral incision sites, allowing insertion of the instrument cannulae into the subplatysmal working space. Next, standard laparoscopic instruments - a suction electrocautery and an ultrasonic-shears - are used to elevate the platysma from the level of the mandible to the sternum inferiorly and laterally to the sternocleidomastoid muscles. Subsequently, and $8-\mathrm{mm}$ bariatric trocar can be inserted through an incision made along the patient's right axillar fold into the subplatysmal working space for countertraction of the strap muscles or perineural tissue during the operation and for insertion of the closed suction drain at the end of the surgery, if desired. In order to create a larger working space, applying few vicryl stitches can help superior retraction of subplatysmal flap. ${ }^{20}$

\section{Docking stage}

After forming a appropriate working space, the robotic system is installed. The cannulae are docked into the robotic arms, starting with the central cannula to secure the position of the endoscope $\left(30^{\circ}\right.$, down facing). A Maryland dissector and the Harmonic scalpel are inserted into the left and right ports, respectively. ${ }^{20}$

\section{Console stage}

Midline raphe is dissected to separate the strap muscles. The strap muscles are dissected from the thyroid gland, and the targeted lobe(s) is exposed. If the pyramidal lobe is identified, the lobe is dissected from the thyroid cartilage, and isthmectomy is performed subsequently. ${ }^{20}$ Once the thyroid lobe is freed from the trachea medially, the superior pole is lifted. Superior lobe is carefully dissected by ligating one vessel at a time. The superior parathyroid gland is identified and preserved first. Then the thyroid lobe is retracted inferiorly to facilitate the identification of the recurrent laryngeal nerve (RLN) at its entry point into the larynx around cricothyroid articulation. ${ }^{7}$ Once the RLN is identified and carefully preserved, the Berry's ligament is localized. To identify and preserve the inferior parathyroid gland, further dissection is done inferiorly. After the inferior lobe is freed off from its surrounding soft tissue, hemithyroidectomy is complete. ${ }^{20}$ The contralateral lobectomy can be done at the same time if necessary, without any additional incision.

Ipsilateral dissection of central compartment with tracing the RLN into the thoracic inlet may be followed if necessary, and the lympho-adipose tissue in the central compartment can be resected en-bloc with the targeted thyroid lobe. ${ }^{20}$ The nerve stimulator probe is utilized to stimulate the RLN and to examine neurophysiologic integrity during and after the procedure. Then, through additional axillar incision, the specimen in an endoscopic bag can be pulled out from the operation field, mostly without any disruption. Meticulous hemostasis and wound irrigation are done. Through the axillary trocar site, a Jackson Pratt drain is inserted and located at the middle raphe of the strap muscles. Then, the muscles are re-
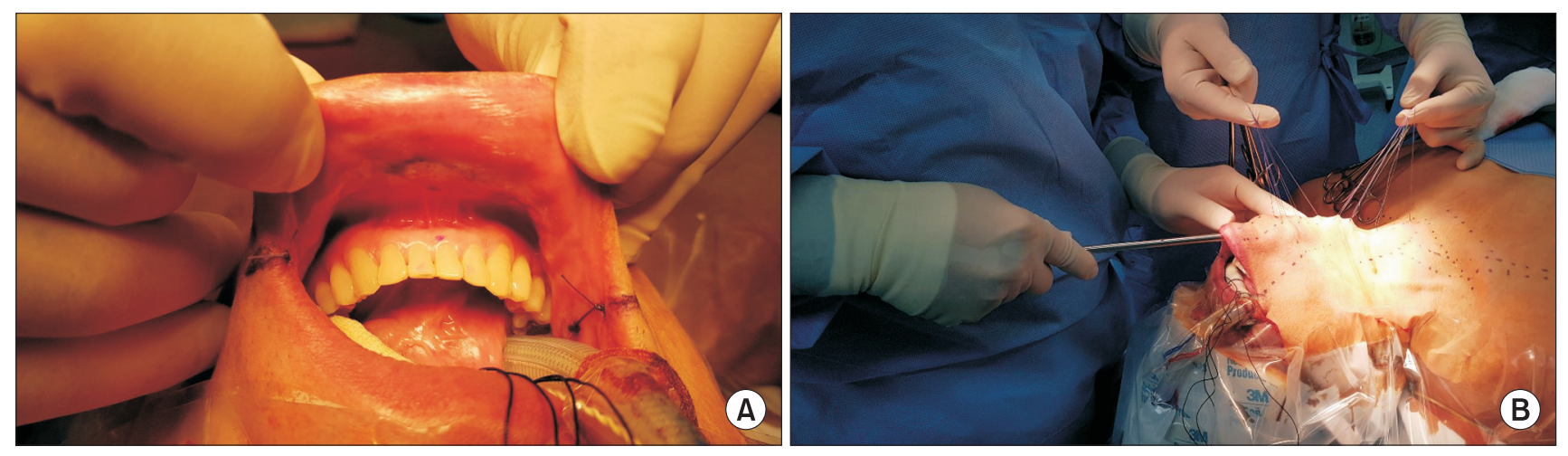

Fig. 1. (A) Location of incisions. (B) Blunt dissection with $8 \mathrm{~mm}$-tipped vascular surgical tunneler. 
approximated with a self-locking absorbable suture. The oral vestibular incisions are closed layer by layer with absorbable sutures. A compression dressing is placed across the neck and chin after the patient has been extubated.?

\section{Postoperative management}

Four hours after the surgery, sips of water is permitted to patients. In the evening on the day of surgery, soft bland diet and ice cream are recommended to the patients, and right on the next day, and normal regular diet can be permitted. Two weeks after the surgery, intraoral stitches are removed at the outpatient clinic.

\section{DISCUSSION}

There has not been enough time to assess the results of TORT because only small number of studies are publihsed in short period of time. The new robotic platform, the da Vinci Xi (surgical intuitive, Sunnydale, CA, USA), has shown advancement of robotic technologies for thyroid surgery, and we must seize this chance to develop transoral, robotic thyroidectomy.

Endoscopic approach reduces working time and workspace, as well as collision of instruments, limitation of movements and difficult displays, which inevitably leading to highly complex procedures and requiring more elaborate surgical techniques.

Considering these limitations, to yield better performance, the evolution of transoral robotic approach should be aimed in five main prospects: trocars, instruments, tremor, and contraction mechanisms, and display. ${ }^{8}$ Another important advance in the robotic thyroidectomy is the introduction of modified curved instruments, which became articulate and also flexible, therefore supporting the proper triangulation and mobility at the operation field. The surgical system Da Vinci Xi offers the wide range of movements and this is widely known advantage that can theoretically reduce crashes among the instruments.

However, this advantage is not fully satisfied or justified. The da Vinci system is too large in size and volume, and its initial settings have not been formulated for many procedures yet. In spite of these drawbacks, surgeons have done transoral thyroidectomies with the da Vinci System, and have proved that it is feasible and safe, standardized the technique. Also, after exploring different surgical approaches: transaxillar, transareolar, retroauricular, the TORT has become popular even with changes of platform. ${ }^{7,8}$

Kim et al. ${ }^{5}$ first reported that the technique is feasible and safe for selected patients after technical refinements, and can be a potential alternative approach for scarless thyroid sur- gery. They described results from the transoral robotic thyroid surgery. ${ }^{5}$ The Author used three intraoral ports and a single axillary port with the system's four robotic arms. The study included twenty-four female patients (mean age 39.6 \pm 11.6 years; mean tumor size $1.0 \pm 1.3 \mathrm{~cm}$ ), who underwent unilateral thyroid lobectomies with or without ipsilateral central neck dissection. Twenty patients had papillary thyroid carcinomas (PTC), three had benign nodules, and one had a follicular thyroid carcinoma. The mean surgical time was $232 \pm 41 \mathrm{~min}$; the mean hospital stay was $3.3 \pm 0.8$ days. The number of retrieved

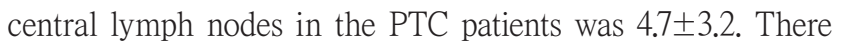
was no transient or permanent vocal cord palsy, recurrence, or mortality during the median follow-up period of 16.8 months. Paresthesia of the lower lip and the chin due to mental nerve injury was observed in first nine patients (six transient patients, three permanent patients). But there was no further report of paresthesia recorded after the first 12 patients, after the locations of the intraoral incisions were modified. ${ }^{5}$

In one recent study, authors claimed that in selected patients, the TORT can safely done and showed equivalent results with Bilateral Axillo-Breast Approach (BABA) robotic thyroidectomy. ${ }^{2}$ A single surgeon's initial surgical outcomes of TORT were compared to those of BABA robotic thyroidectomy. Each group was composed of 50 patients. In transoral robotic total thyroidectomy group, operative times were shorter, pain scores were lower, and hospital stays were shorter than BABA robotic thyroidectomy group. As in rates of vocal cord palsy and hypoparathyroidism, there was no significant difference between the two groups. In first 12 cases of TORT, 9 cases of mental nerve injury occurred, but no such cases occurred subsequently. ${ }^{2}$

Recently, surgical outcomes of 372 TORT cases were announced. Only one case was converted to open thyroidectomy due to a huge goiter. The operation time became shorter to average 199.3 minutes. The number of retrieved central lymph nodes was increased to mean 5.14. The new complication was happened - one hematoma, one chyle leakage, one localized infection - these were well treated (Table 2).

\section{Advantages}

Definitely, cosmesis is the major advantage of transoral thyroidectomy. Transoral thyroidectomy is cosmetically superior to any other approaches because intraoral incisions heals in 2 to 3 weeks and becomes completely invisible in a few months. ${ }^{5}$ Extensive flap dissection is not required in transoral thyroidectomy. While gasless transaxillary and bilateral axillo-breast approach requires additional wide flap dissection at axilla and upper chest respectively, extent of dissection in transoral thyroidectomy is much smaller than the other two approaches. 
Table 2. Surgical outcomes in recent TORT 372 cases

\begin{tabular}{|c|c|}
\hline Variables & Value $(n=372)$ \\
\hline \multicolumn{2}{|l|}{ Operation method } \\
\hline Lobectomy & $37(9.9 \%)$ \\
\hline Lobectomy+CND & $308(82.9 \%)$ \\
\hline Total thyroidectomy + CND & $22(5.9 \%)$ \\
\hline Bilateral subtotal thyroidectomy & $3(0.8 \%)$ \\
\hline Total thyroidectomy + CND + RLND (level III, IV) & $2(0.5 \%)$ \\
\hline Operation time (mean $\pm S D$ ), min & $199.3 \pm 39.2$ \\
\hline Conversion to open conventional surgery & $1(0.2 \%)$ \\
\hline \multicolumn{2}{|l|}{ Pathologic diagnosis } \\
\hline Benign & $36(9.7 \%)$ \\
\hline Follicular thyroid carcinoma & $5(1.3 \%)$ \\
\hline Papillary thyroid carcinoma & $330(89.0 \%)$ \\
\hline \multicolumn{2}{|l|}{ Tumor size (mean $\pm S D$ ), cm } \\
\hline Benign & $2.71 \pm 1.01$ \\
\hline Malignant & $0.77 \pm 0.48$ \\
\hline \multicolumn{2}{|l|}{ Nodal stage in papillary carcinoma patients $(\mathrm{n}=330$ ) } \\
\hline NO & $188(57.0 \%)$ \\
\hline N1a & $139(42.1 \%)$ \\
\hline $\mathrm{N} 1 \mathrm{~b}$ & $3(0.9 \%)$ \\
\hline Number of retrieved central LNs (mean \pm SD) & $5.14 \pm 4.2$ \\
\hline \multicolumn{2}{|l|}{ Complications } \\
\hline Hematoma (Undergone operative hemostasis) & $1(0.3 \%)$ \\
\hline Seroma or Fluid collection & $0(0 \%)$ \\
\hline Chyle leakage & $1(0.3 \%)$ \\
\hline Infection, localized or systemic & $1(0.3 \%)$ \\
\hline \multicolumn{2}{|l|}{ Vocal cord palsy } \\
\hline Transient & $3(0.8 \%)$ \\
\hline Permanent & $0(0 \%)$ \\
\hline \multicolumn{2}{|l|}{ Hypoparathyroidism } \\
\hline Transient & $1(4.5 \%$, out of 22$)$ \\
\hline Permanent & 0 (0\%, out of 22) \\
\hline Mental nerve injury & $9(2.4 \%)$ \\
\hline Stretching & $6(1.6 \%)$ \\
\hline Tearing & $3(0.8 \%)$ \\
\hline Bruise on zygoma & $2(0.5 \%)$ \\
\hline Bruise on flap & $3(0.8 \%)$ \\
\hline Chin flap perforation & $2(0.5 \%)$ \\
\hline Oral commissure tearing & $2(0.5 \%)$ \\
\hline Skin dimpling in midline of lower chin & $2(0.5 \%)$ \\
\hline Hematoma in intraoral trocar insertion sites & $2(0.5 \%)$ \\
\hline Skin flap burn & $2(0.5 \%)$ \\
\hline
\end{tabular}

The extent is similar to that of conventional open thyroidectomy: covering sternal notch to thyroid cartilage level. Smaller dissection area can prevent sensory loss or postoperative pain caused by flap dissection, which are significant disadvantages in the other remote-access approaches. ${ }^{20}$

TORT has several other strengths too. Originally, when pyramidal lobe or upper pole of the thyroid is highly or deeply located, complete resection with rigid endoscopic instruments might be difficult because the direction of approach is cephalad to caudal. However, using articulated movement of endowrist, surgeons can approach much deeper and upper part of thyroid more easily through transoral approach. Second, tremor-filtering system of robot enhances fine performance and safe dissection around the critical structures such as parathyroid glands or RLNs. In addition, fourth articulating instrument from the axilla enables the counter-traction of strap muscles or perineural tissue, which ensures the precise and radical dissection around the thyroid tissue. Lastly, whereas monitors of endoscopic thyroidectomy are set relatively far from the surgeon since TORT magnifies the view in threedimension, operative view of TORT is superior to that of endoscopic thyroidectomy. ${ }^{20}$

\section{Disadvantages}

As one of the most threatening complications of TORT, mental nerve injury occurred during first several trials. Mental nerve is a sensory nerve that innervates and the skin of the lower lip and the chin. Therefore, after such injury due to the surgery, patients feels numbness around lower lip and chin. Mental nerve emerges from the mental foramen in the mandible and is divided into upper and lower branches. Mental foramen is generally located below canine root, and mental nerve runs medially after branching. However, considering the anatomy of mental nerve, this complication was resolved by modifying port placement and by avoiding over-stretching of working port. Keeping these in mind, mental nerve injuries did not occur anymore after the initial 12 cases. Therefore, the nerve is less likely to be injured when the ports are not overstretched, and are placed more laterally and more distal to canine root. ${ }^{20}$

Minor complications, such as bruise on zygomatic region and tearing of oral commissures, caused by excessive force of robotic arm movements were observed initially as well. ${ }^{5} \mathrm{How}^{-}$ ever, zygomatic bruise can be avoided by careful monitoring of assistants along with protective sponge application over zygoma. ${ }^{20}$ And continuous sutures to protect around oral commissures with plaster might also prevent the minor complications. $^{5}$ 


\section{CONCLUSIONS}

As one of the most novel approaches, TORT draws attention because of its cosmetic excellence, smaller flap dissection area, and much easier and safer approach to the field of operation. Though the transoral technique may raise the risk of infection in the anterior neck region, animal experiments did not show any severe infections, or seromas after administration of prophylactic antibiotics and insertion of drain.

By overcoming several disadvantages with simple techniques, TORT can be done safely to the appropriately selected patients by surgeon expertise in robotic thyroidectomy. Further research should be targeted towards the expansion of criteria for potential candidates, and also towards the shorter operative time and more comfortable instruments and feasible techniques.

Thus, we can conclude that TORT might be a potential alternative surgical approach for thyroidectomy to surgeons who are experienced in remote-access robotic surgery.

\section{CONFLICT OF INTEREST}

Jeong Min Choo, Ji Young You and Hoon Yub Kim declare that they have no conflicts of interest.

\section{REFERENCES}

1) Dionigi G, Wu CW, Tufano RP, et al. Monitored transoral endoscopic thyroidectomy via long monopolar stimulation probe. J Vis Surg 2018;4:24.

2) Chai YJ, Kim HY, Kim HK, et al. Comparative analysis of 2 ro- $^{-}$ botic thyroidectomy procedures: Transoral versus bilateral axillobreast approach. Head Neck 2018;40:886-892.

3) Dionigi G, Chai YJ, Tufano RP, Anuwong A, Kim HY. Transoral endoscopic thyroidectomy via a vestibular approach: why and how? Endocrine 2018;59:275-279.

4) Chai YJ, Chung JK, Anuwong A, et al. Transoral endoscopic thyroidectomy for papillary thyroid microcarcinoma: initial experience of a single surgeon. Ann Surg Treat Res 2017;93:70-75.

5) Kim HY, Chai YJ, Dionigi G, Anuwong A, Richmon JD. Transoral robotic thyroidectomy: lessons learned from an initial consecutive series of 24 patients. Surg Endosc 2018;32:688-694.

6) Anuwong A, Sasanakietkul T, Jitpratoom P, et al. Transoral endoscopic thyroidectomy vestibular approach (TOETVA): indications, techniques and results. Surg Endosc 2018;32:456-465.

7) Richmon JD, Kim HY. Transoral robotic thyroidectomy (TORT): procedures and outcomes. Gland Surg 2017;6:285-289.

8) Anuwong A, Kim HY, Dionigi G. Transoral endoscopic thyroidectomy using vestibular approach: updates and evidences. Gland Surg 2017;6:277-284.

9) Dionigi G, Lavazza M, Wu CW, et al. Transoral thyroidectomy: why is it needed? Gland Surg 2017;6:272-276.

10) Chang EHE, Kim HY, Koh YW, Chung WY. Overview of robotic thyroidectomy. Gland Surg 2017;6:218-228.

11) Dionigi G, Tufano RP, Russell J, Kim HY, Piantanida E, Anuwong A. Transoral thyroidectomy: advantages and limitations. J Endocrinol Invest 2017;40:1259-1263.

12) Dionigi G, Bacuzzi A, Lavazza M, et al. Transoral endoscopic thyroidectomy: preliminary experience in Italy. Updates Surg 2017;69:225-234.

13) Dionigi G, Lavazza M, Bacuzzi A, et al. Transoral Endoscopic Thyroidectomy Vestibular Approach (TOETVA): From A to Z. Surg Technol Int 2017;30:103-112.

14) Russell JO, Noureldine SI, Al Khadem MG, et al. Transoral robotic thyroidectomy: a preclinical feasibility study using the da Vinci Xi platform. J Robot Surg 2017;11:341-346.

15) Dionigi G, Bacuzzi A, Lavazza M, et al. Transoral endoscopic thyroidectomy via vestibular approach: operative steps and video. Gland Surg 2016;5:625-627.

16) Clark JH, Kim HY, Richmon JD. Transoral robotic thyroid surgery. Gland Surg 2015;4:429-434.

17) Lee HY, Richmon JD, Walvekar RR, Holsinger C, Kim HY. Robotic transoral periosteal thyroidectomy (TOPOT): experience in two cadavers. J Laparoendosc Adv Surg Tech A 2015;25:139-142.

18) Lee HY, You JY, Woo SU, et al. Transoral periosteal thyroidectomy: cadaver to human. Surg Endosc 2015;29:898-904.

19) Razavi CR, Fondong A, Tufano RP, Russell JO. Central neck dissection via the transoral approach. Ann Thyroid 2017 Oct 31 [Epub]. DOI: 10.21037/aot.2017.10.02.

20) Kim HY. Transoral Robotic Thyroidectomy. Current Surgery Reports 2017;5:19. 